\title{
La perspectiva generacional en los estudios de juventud: enfoques, diálogos y desafíos
}

Carolina Álvarez Valdés'

\section{RESUMEN}

El presente artículo es una revisión del tratamiento de la perspectiva generacional en los estudios de juventud, con énfasis en lo que ha sido publicado en Revista Última Década y sus autores principales. A partir de lo cual se identificaron dos enfoques predominantes: culturalista e histórico-sociológico. Los resultados señalan que ambos enfoques tienen autores clásicos a la base, con una preponderancia de la conceptualización desarrollada por Mannheim, aun así es posible identificar la influencia de Ortega y Gasset, principalmente en el enfoque culturalista. El enfoque culturalista comprende a las generaciones como referentes simbólicos, son centrales los procesos de globalización y los cambios culturales. Mientras que, el enfoque histórico-sociológico se ha centrado en el estudio de trayectorias juveniles y de militancias políticas, con una preferencia por el estudio de grupos concretos en contextos locales y nacionales, con un desarrollo mayor de trabajos empíricos. Los desafíos de esta perspectiva es avanzar en la producción de mayor investigación social, que permita actualizar las referencias teóricas clásicas y las metodologías utilizadas.

PALABRAS CLAVE: GENERACIONES, ESTUDIOS DE JUVENTUD

1 Doctora @ en Educación (Universidad Alberto Hurtado/Universidad Diego Portales, Santiago, Chile). Integrante del Núcleo Investigación y Acción en Mundos Juveniles, Facultad de Ciencias Sociales (Universidad de Chile). Correo electrónico: caroalvarezvaldes@gmail.com. 


\title{
A perpectiva geracional nos estudos de juventude: abordagens, diálogos e desafios
}

\section{RESUMO}

Este artigo é uma revisão do tratamento da perspectiva geracional nos estudos de juventude, com ênfases no que foi publicado na Revista Última Década e seus principais autores. A partir do qual foram identificadas duas abordagens predominantes: culturalista e histórico - sociológica. Os resultados mostram que as duas abordagens têm autores clássicos à base, com uma preponderância da conceptualização desenvolvida por Mannheim, inclusive assim é possível identificar a influência e Ortega y Gasset, principalmente na abordagem culturalista. Esta última abordagem inclui as gerações como referentes simbólicos e os processos de globalização e as mudanças culturais são centrais. Enquanto, a abordagem histórico-sociológica se focalizou no estudo das trajetórias juvenis e das militâncias políticas, com uma preferência pelo estudo de grupos concretos em contextos locais e nacionais, com maior desenvolvimento de trabalhos empíricos. Os desafios desta perspectiva são avançar na produção de mais pesquisa social, que permita atualizar as referências teóricas clássicas e as metodologias utilizadas.

\section{The generational perspective in youth studies: approaches, dialogues and challenges}

\begin{abstract}
The present article is a review of the treatment of generational perspective in youth studies, focused on the articles published on Revista Última Década and its main authors; two main approaches, culturalist and historical-sociological, have been identified from such review. The results characterize both approaches as based on classical authors, with a prominent presence of the conceptualization developed by Mannheim, not ignoring Ortega y Gasset's influence, mainly on the culturalist approach. The latter approach understands generations as symbolic referents, with globalization processes and cultural changes as central aspects. The historical-sociological approach, on the other hand, is centered on the study of youth trajectories and political affiliations, focusing on the study of determined groups within national and local contexts and with further development of empirical studies. The challenge of this perspective is
\end{abstract}


to move forward in the production of more social research in order to update the classic theoretical references and used methodologies.

KEYWORDS: GENERATIONS, YOUTH STUDIES

\section{INTRODUCCIÓN}

Los estudios generacionales han intentado responder a la relación existente entre la renovación biológica y vital de los seres humanos y la construcción histórica de las sociedades, buscando una clave de análisis para la comprensión del cambio social (Muñoz, 20Irb). Es una línea de investigación que se ha retomado en los últimos años en los estudios de juventudes en Chile, América Latina y países anglófonos, ya que permite historizar en cada cultura y época las relaciones inter e intra generacionales junto con acercarse a la comprensión de las juventudes desde una conceptualización contextual, mirando lo social desde lo juvenil, por medio de la articulación entre el tiempo biográfico y el tiempo histórico (Aguilera, 20I4; Canales, Ghiardo \& Opazo, 20I5; Duarte, 2005, 20I5; Ghiardo, 2004; González y Feixa, 2013; Leccardi y Feixa, 20II; Feixa, 2006, 2000; Madrid, 2005; Muñoz, 20Ira, 20IIb).

Los trabajos en Chile de esta perspectiva, se han centrado, más bien, en entender fenómenos políticos, que tienen relación con la juventud, en tanto, se entiende este período de la vida como eje clave en la socialización política, en términos de vivencias que definen militancias y participación política, a propósito de un contexto particular y de experiencias fundantes para los sujetos (Baño y Faletto, I992; Contreras y Navia, 2013; Madrid, 2005; Muñoz, 20ıra, 2OIIb).

En este artículo, propongo seguir una ruta de lectura sobre la perspectiva generacional, a partir de la producción de Revista Última Década (RUD), a propósito del aniversario de sus 25 años y de su especificidad y centralidad en los estudios de juventud. Si bien esta revista es chilena, alberga trabajos de distintos países y continentes, con predominancia de estudios nacionales. No obstante, la revisión de artículos sobre generaciones en RUD es un mapa sobre esta producción, tanto en Chile como en América Latina.

En este trabajo de revisión se apuesta a identificar tendencias, teorías, conceptos y metodologías predominantes en las distintas corrientes y autores. Para luego ponerlas en diálogo con producciones académicas desarrolladas en distintos países, buscando desbordar la propia producción de la revista. Pues, en el contexto internacional, también se ha retomado esta perspectiva, en el marco de las experiencias y condiciones actuales de la sociedad y los jóvenes, a propósito del escenario neoliberal y de los procesos de globalización, que han implicado una serie de cambios en distintos ámbitos: educación, trabajo y las 
transiciones desde la educación al mundo del trabajo, comunicación, referentes culturales y simbólicos, relaciones sociales, entre otros. Produciéndose una relación entre biografías y condiciones estructurales que podrían ser comprendidas desde el marco conceptual generacional.

La perspectiva generacional es hasta hoy un terreno en disputa, pues continúan siendo problemáticas cuestiones tales como: ¿qué define a una generación?, ¿cuándo podemos hablar de una generación?, ¿cuáles son sus límites?, ¿dónde comienza una generación y termina otra? Estas preguntas encuentran distintas respuestas, dependiendo de los enfoques y epistemes de los trabajos revisados.

\section{LA PERSPECTIVA GENERACIONAL: ENFOQUES Y DIÁLOGOS POSI- BLES}

En la revisión de las publicaciones de RUD, es posible observar que la perspectiva generacional, tal como en el contexto internacional y nacional, es de reciente interés. El primer trabajo que considera su pertinencia para estudiar a las juventudes es del año 2004 (Ghiardo, 2004), le sigue un período sin producción sobre la temática, para volver recién a partir del año 20II, fecha que marca una mayor sistematicidad en la producción de artículos que abordan la perspectiva generacional teórica y/o empíricamente. De hecho, entre los años 20II y 20I3 se sitúan la mayor cantidad de trabajos en RUD sobre la temática (Leccardi \& Feixa, 20II; Duarte, 20I2; Portillo et al, 20I2; Dávila \& Ghiardo, 20I2; Muñoz, 20II; Canales \& Ghiardo, 20I2; Infantino, 2013).

Cabe preguntarse, si esta producción más sistemática, entre los años $201 \mathrm{I}$ y 20I3, responde a cierto contexto particular, que hace necesario volver la mirada sobre esta perspectiva. En este sentido, el año 201 i parece ser clave, es posible fechar varios sucesos de protestas a nivel global, donde los y las jóvenes fueron protagonistas: el movimiento indignados ${ }_{5} \mathrm{M}$ en España, Occupy de Wall Street, las revueltas árabes y en Chile el movimiento estudiantil. ¿Por qué estos sucesos nos hacen volver a la pregunta por las generaciones? Una posible respuesta es que la perspectiva generacional es una manera de comprender las transformaciones sociales, la renovación de las sociedades y su relación con las juventudes en distintos contextos.

Esta producción más sistemática que aborda la perspectiva generacional, ya sea como clave de lectura de la sociedad y las juventudes y/o como profundización teórica, tiene elementos teóricos y metodológicos, con algunas similitudes y diferencias en sus formas de comprender las generaciones y su estudio. Así es como a partir de la revisión de trabajos de RUD se pueden agrupar en dos grandes enfoques y epistemes la perspectiva generacional: culturalista e histórico-sociológico. El enfoque culturalista, desarrollado principalmente por antropólogos/as, comprende las generaciones en términos más bien simbólicos, atravesadas por cambios culturales profundos y globales. Mientras que el 
segundo enfoque, desarrollado por historiadores y sociólogos, comprende a las generaciones en términos históricos y situados en contextos locales y nacionales, por lo mismo, estudian más bien grupos concretos de sujetos. Previo de la presentación de estos enfoques se hace necesaria una revisión de los autores a la base, referenciados transversalmente por los trabajos de ambos enfoques.

\section{LAS GENERACIONES EN ORTEGA Y GASSET Y MANNHEIM: UNA REVISIÓN NECESARIA}

Antes de comenzar con la revisión de autores contemporáneos que han abordado la temática generacional, ya sea en una vertiente teórico y/o empírica, es necesario revisar a dos autores que están a la base de los enfoques identificados. Lo que además representa una decisión práctica, ya que a lo largo del texto haré referencia a conceptos e ideas abordadas por estos autores. Primero, me detendré en la obra de Ortega y Gasset (1966; 1970) sobre generaciones, autor que, desde la filosofía, desarrolla una visión anclada en las edades como explicación del cambio social, las generaciones serían una clave de comprensión del ritmo de la historia. Posteriormente, ahondo en la conceptualización elaborada por Mannheim (1993) sobre las generaciones, quien desarrolla una serie de nociones que permitirían estudiar a las generaciones como un problema sociológico. Aporte que es considerado transversalmente en los distintos enfoques y modos de abordar esta perspectiva, algunos destacan ciertos conceptos sobre otros, lo que marca diferencias en el acercamiento conceptual y empírico.

\section{EL RITMO DE LA HISTORIA: LAS GENERACIONES EN ORTEGA Y GAS- SET}

A partir de la historia del pensamiento filosófico, Ortega y Gasset (ig66) sostiene, que los colectivos sociales pueden tomar dos posiciones respecto al pensamiento presente: épocas donde el pensamiento se considera como parte del desarrollo previo, mientras en otras épocas consideran que deben cambiar el pensamiento del pasado desde la raíz. Unos serían los tiempos de la filosofía pacífica y otros de la filosofía beligerante. Cuando son tiempos beligerantes, el colectivo se separa en dos, la masa que defiende lo establecido y un pequeño grupo de vanguardia que debe defenderse de aquellos que no ven las transformaciones venideras. Así es como las variaciones en la sensibilidad vital (mundo vigente), que son decisivas, serían una generación, que está compuesta de esta minoría selecta y la muchedumbre. Para Ortega y Gasset (I966) el concepto de generación, es el más importante para la historia, "el gozne sobre que ésta ejecuta sus movimientos" (p.I47). Ortega y Gasset (I966) reconoce la conflictividad dentro de una misma generación, pero más allá de existir posturas contrapuestas, los sujetos tienen mayor afinidad por ser parte de un mismo tiempo.

En un primer trabajo Ortega y Gasset (1966) plantea que las generaciones 
nacen las unas de las otras, deben recibir el pasado en el presente, pero pueden crear algo distinto con lo que recibieron. Posteriormente, señala que más que sucederse unas de otras, se empalman o solapan, siempre dos generaciones protagonistas actúan al mismo tiempo. Cuestión que se explica por la definición de clases de edad que realiza: niñez, juventud, iniciación, predominio y vejez. Etapas que van de I5 en I5 años, y cada una tiene un quehacer particular. Las edades más relevantes son las de iniciación y predominio. Estas dos generaciones -iniciación (30 a 45 años) y predominio ( 45 a 60 años)- coexistirían en un mismo tiempo histórico, con distinto sentido, debido a la diferencia de edad que tienen y sus quehaceres correspondientes (Ortega y Gasset, I970):

[Desde los 30 años] el hombre comienza a reaccionar por cuenta propia frente al mundo que ha hallado, inventan nuevas ideas sobre los problemas del mundo (...) Y así, un buen día, se encuentran que su mundo innovado, el que es obra suya, queda convertido en mundo vigente (...) En ese momento empieza una nueva etapa de la vida: el hombre sostiene el mundo que ha producido, lo dirige, lo gobierna, lo defiende. Lo defiende porque unos nuevos hombres de treinta años comienzan, por su parte, a reaccionar ante ese nuevo mundo vigente. (p.47)

Ortega y Gasset (I970) propone un método de las generaciones en la historia, que tiene dos elementos claves, por un lado, más que la fecha de nacimiento es la "zona de fechas" que ha determinado en I5 años. Por otro lado, la manera de definir a los individuos dentro de una generación, es por medio de la determinación de la fecha de nacimiento de una generación, a partir de la identificación de una figura que represente los caracteres sustantivos del período que se erige como nuevo, y reconociendo la fecha en la que cumple 30 años, edad en que comienza a desarrollar este nuevo mundo, como señala la cita previa (I970).

\section{EL PROBLEMA DE LAS GENERACIONES EN MANNHEIM}

Un segundo autor citado transversalmente dentro de la perspectiva generacional es Karl Mannheim, quien en clásico ensayo "El problema de las generaciones" (I993) elabora una conceptualización multidimensional (Muñoz, 20Ira), desarrollando las siguientes nociones: posición generacional, conexión generacional, unidad generacional y estratificación de la vivencia; que permitirían comprender la formación de una generación (Mannheim, I993).

La posición generacional se fundamenta en la existencia de un ritmo biológico; en hechos como la vida, la muerte y la edad. Los sujetos tienen una posición generacional similar a la de otros por compartir una época cercana de nacimiento y con ello ser parte de una corriente histórica con determinadas formas de vivencia y pensamiento. Asimismo, los sujetos deben compartir una comunidad de vida histórica (ser parte de una misma sociedad) (Mannheim, r993). Mientras que la conexión generacional da cuenta del vínculo entre los individuos de una 
posición generacional con los contenidos del momento histórico que están viviendo, en la medida que toman parte activa o pasiva en aquellas interacciones que conforman la nueva situación (Mannheim, 1993). Es posible que dentro de una misma conexión generacional se den ideas e interpretaciones opuestas sobre una misma problemática histórica, estas oposiciones dan lugar a distintas unidades generacionales. Estas unidades son los que vinculan a los individuos con el grupo porque tienen efecto socializador, comparten ciertos contenidos. Las unidades implican un modo de reaccionar unitario, "un agitarse juntos" (Mannheim, I993, p. 225).

La estratificación de la vivencia está vinculada con la formación de la conciencia, para lo cual resultan decisivas las vivencias que se constituyen como primeras impresiones, las vivencias de juventud, y aquellas que vienen en segundo, tercer orden y así sucesivamente. Es fundamental para los individuos el hecho de que opere una decisiva primera impresión de juventud, o que no lo haga y funcionen como una vivencia tardía. Así, las experiencias posteriores o tardías, estarían orientadas por aquella primera impresión, ya sea a través de la negación o afirmación. No se acumulan simplemente experiencias en el curso de vida, sino que se articulan de manera dialéctica (Mannheim, 1993).

Dos generaciones distintas en el tiempo se enfrentan a mundos exteriores e interiores distintos. Una generación se enfrentó a un contexto (estructuras) y en él obtuvo las primeras impresiones del mundo, en la siguiente generación ese contexto cambió por lo que sus primeras impresiones son distintas, y sin embargo, ese contexto que cambió aún permanece en la generación anterior. De esta manera, lo que para una generación no fue problemático sí lo es para la otra (Mannheim, i993). Esto último, explicaría la conflictividad con que jóvenes analizan y actuán en el presente.

\section{ENFOQUE CULTURALISTA}

El enfoque culturalista, dentro de la perspectiva generacional, agrupa a autores $\mathrm{y}$ trabajos que comprenden a las generaciones como referentes culturales, en una doble lógica: por un lado, las generaciones determinadas por un contexto particular como la globalización y lo que esta trae consigo, como uso intensivo de las tecnologías de la información, y los cambios culturales en las formas de ser jóvenes en las sociedades contemporáneas -se relevan procesos de migración e hibridez cultural); por otro lado, comprende a las generaciones como productoras de una cierta cultura, es decir, toman elementos del mundo que reciben pero crean algo distinto con él. Se entiende este juego como una relación dialéctica, donde los y las jóvenes son producidos y a la vez, van desplegando sus propias maneras de pensar, actuar y sentir en la sociedad actual.

En el enfoque culturalista, una generación es un referente simbólico, tal como plantea Feixa: "las generaciones no son estructuras compactas, sino sólo referentes simbólicos que identifican vagamente a los agentes socializados en 
unas mismas coordenadas temporales". (Feixa, 200o, p. 87). Serían, entonces, agrupaciones identitarias, que comparten cercanía en su fecha de nacimiento y un contexto común, marcado por ciertos hitos.

Leccardi \& Feixa (20II), inauguran el período de mayor sistematicidad en el desarrollo de esta perspectiva en RUD, realizan una revisión del uso del concepto generación, centrando su análisis en las raíces del concepto en las teorías de juventud, específicamente en Italia y España, países de origen de los autores. Comienzan su trabajo con una lectura del desarrollo teórico sobre generaciones en la sociología, deteniéndose en los aportes de Comte, Dilthey, Mannheim y Abrams. Identifican en Mannheim un punto de inflexión, ya que se distancia de las tradiciones previas sobre la temática, positivistas y romántico-historicistas, representadas por Comte y Dilthey, respectivamente. Para Leccardi \& Feixa (20II), Mannheim Abrams tienen puntos de coincidencia, en tanto, sería el tiempo histórico y los cambios sociales los que definirían a las nuevas generaciones y sus identidades. Además reconocen en España el fuerte influjo de la obra de Ortega y Gasset y la vuelta de esta perspectiva desde la década de los 80 .

Ahora, sobre las maneras de comprender a una generación, Leccardi \& Feixa (20II) introducen el concepto de conciencia generacional, desarrollado en Italia, que refiere a la historicidad y su vínculo con la experiencia. Respecto a este punto, abordan un elemento interesante a considerar dentro de la perspectiva generacional, que es la capacidad de los sujetos de situarse históricamente, en relación a generaciones previas y futuras, y la elaboración subjetiva sobre esto. Junto con ello, pone en el centro la transmisión intergeneracional, es decir, que dentro de las familias, se traspasa una cierta concepción natural del mundo, de manera no necesariamente intencional ni reflexiva.

De acuerdo a Leccardi \& Feixa (20II), la perspectiva generacional sigue vigente, aunque no ha renovado sus bases teóricas ni metodológicas. Plantean como punto de salida a esta perspectiva en la actualidad, el concepto de generación global, específicamente la idea de constelaciones generacionales cruzadas, que analizan los factores universales que afectan a las generaciones. A partir de esta conceptualización Leccardi y Feixa (20II) identifican factores cosmopolitas de la generación actual: generación de la migración, generación aprendiza (empleo precario) y generación patchwork, relacionada con las marcas de la hibridación cultural. Además se hace referencia a un nuevo contrato social entre generaciones mayores y jóvenes, por un lado trae complicaciones en el mercado del trabajo, las pensiones, etc. Pero por otro, hace referencia a nuevos lazos solidarios, reflejado en un alto grado de reciprocidad entre generaciones (padres e hijos).

El trabajo de Leccardi \& Feixa (20II), constituye un esfuerzo por sistematizar las teorías clásicas y actuales. No obstante, queda como desafío el desarrollo de una propuesta propia a partir de la exhaustiva revisión que realizaron y sobre todo del contexto actual para las juventudes, que identifican ampliamen- 
te y de manera global con el concepto de constelaciones generacionales cruzadas, pero que no tiene referencia empírica ni avizora cuestiones teórico metodológicas a ser abordadas en futuras investigaciones.

El artículo de Leccardi \& Feixa (2OII) es parte de la vasta obra de este último autor, (Feixa, 2000; 2006; González \& Feixa, 20I3; Leccardi \& Feixa 20II), quien se constituye en un referente dentro de esta perspectiva. Feixa, es doctor en antropología de la Universidad de Barcelona, autor de numeros libros y artículos que abordan el estudio de las juventudes, teórica y empiricamente, sus estudios han estado centrados en las culturas juveniles, en España y México. Ahora, en el trabajo que ha desarrollado respecto a las generaciones, la conceptualización elaborada ha sido pensada principalmente a partir de la realidad europea y norteamericana, haciendo guiños a Latinoamérica (González \& Feixa, 2013; Feixa, 2006).

Feixa (2006) comprende el devenir del siglo XX - europeo y norteamericano- como la sucesión de generaciones de jóvenes, que desde inicios de siglo comienzan a desplegar una cultura distintiva a la de los adultos. Para ello, denomina a las generaciones de acuerdo a las distintas décadas que la componen. En la definición que realiza sobre las generaciones, va identificando hitos históricos y culturales en relación a la manera de conceptualizar al sujeto joven por parte de las ciencias sociales. Cada generación la cataloga con una letra, generación A, B, K, S, E, R, H, P, T, R. Este autor comprende a las generaciones como referentes simbólicos- no como grupos concretos a ser estudiados con metodologías ad hoc- definidas por una cierta cercanía en la fecha de nacimiento y por vivenciar ciertos hitos históricos de manera conjunta, no definidas tampoco en términos territoriales, excede lo local y nacional. En este sentido, es posible encontrar algunas similitudes con la obra de Ortega y Gasset (1966; 1970), sobre todo en la búsqueda de comprender la historia como la sucesión de generaciones y de rotularlas cada io años con una letra que sería una metáfora de las juventudes de ese período - en Ortega y Gasset eran diferencias de I5 años-. No se explicita el modo en el que se determinó las distintas letras para cada generación, pues en algunos casos son eventos políticos y en otros culturales.

En un texto posterior de Portillo, Urteaga, González, Aguilera \& Feixa (2012), publicado en RUD y parte de la perspectiva culturalista, con un núcleo teórico anclado en los postulados de Feixa- quien es uno de los autores -, revisan cinco experiencias de jóvenes latinoamericanos, específicamente en México y Chile, en ámbitos identitarios y políticos, para poner en discusión la existencia de una generación X y @ en América Latina. En este sentido, los hallazgos señalan un empalme de las generaciones X y @ - pensadas para Europaen América Latina, pues los tiempos sociales serían distintos en este lado del globo, marcados por otros procesos socio-históricos, tales como las dictaduras militares.

Es posible entonces, ver una continuidad en la obra de Feixa (2006) en este trabajo conjunto con autores latinoamericanos, cercanos a la antropología, 
pero esta vez los rótulos son otros, X y @. La generación X la componen los jóvenes de la década de los 9o's, marcados por las incertidumbres, por la falta de un sistema de valores único y sólido. Mientras que la generación @ sería aquella juventud que nació a finales del siglo XX, y se expresa en tres tendencias de cambio: acceso universal a las nuevas tecnologías de la información, difuminación de los límites entre sexos y géneros y la globalización cultural que trae aparejada nuevas formas de exclusión. A partir de los desfases tecnológicos en América Latina, respecto a los países occidentales desarrollados - que es donde fueron pensadas estas categorías- es que se dan estos empalmes entre la generación X y @ (Portillo et al 2012).

Portillo et al (2012) reconocen en el artículo varios elementos que pueden ser cuestionables, pero aun así, no quedan resueltos en el mismo. Señalan que las categorías son pensadas para otros contextos, que no se busca homogeneizar a los y las jóvenes con rótulos y con el simple hecho de nombrarlas. No obstante, se insiste en afirmar el tránsito entre la generación X y @, que es una manera de catalogarlas y nombrarlas. Junto con lo anterior, los autores advierten la diversidad de contextos socioculturales presentes en América Latina. Para dar cuenta de esto, se revisan 5 experiencias de jóvenes latinoamericanos en distintos contextos y con diferentes énfasis, que no responden necesariamente a la conceptualización de generación como referentes simbólicos - véase trabajos de González y Aguilera dentro del mismo artículo-. A partir de esta revisión de experiencias se identifican 5 tendencias centrales en este empalme generacional, que podrían ser cuestiones que no necesariamente responden sólo a este tránsito, tales como la desigualdad social que marca las experiencias juveniles; la interculturalidad; la tensión rural, indígena y urbano; que parecen más bien cuestiones transversales a las experiencias juveniles en América Latina durante el siglo XX. ${ }^{2}$

No obstante lo anterior, Feixa en un trabajo en conjunto con Fernández-Planells y Figueroas-Maz (Fernández-Planells, Feixa, \& Figueroas-Maz, 2013), estudian el movimiento I5- $\mathrm{M}^{3}$ en Barcelona, el perfil de quienes son parte de éste y sus formas de comunicación. Para lo cual despliegan una serie de instrumentos metodológicos, tales como observación participante, encuestas, entrevistas en profundidad y análisis de Twitter. Este trabajo se sitúa teórica-

2 Un tercer trabajo identificado en este enfoque es de Infantino (2013) quien estudia lo generacional a partir de la realización de una etnografía con jóvenes artistas circenses en Buenos Aires. Tiene como base teórica la conceptualización desarrollada por Feixa, pero se diferencia en tanto estudia un grupo concreto por medio de metodología cualitativa. Por lo mismo, identifica dinámicas internas y propias del grupo estudiado en relación al contexto social más amplio argentino.

3 El 15-M también conocido como movimiento de indignados, se llamó así al movimiento de jóvenes en España que protestó contra el sistema político imperante, apostando por mejorar el sistema democrático. El día 15 de mayo del año 2011 se hace ocupación de la Puerta del Sol en Madrid y el 16 de mayo se ocupa la Plaza de Catalunya en Barcelona. Comienza una escalada de manifestaciones en toda España, se hace ocupación de plazas en distintas ciudades. 
mente en la literatura sobre movimientos sociales, destacando elementos nuevos y de continuidad en el $\mathrm{I}_{5}$-M de Barcelona. Sin embargo, en la discusión final, se preguntan por la constitución de una generación a partir del I 5 -M, por la crisis del contexto y la respuesta de este movimiento. En este sentido, se ve una diferencia con los trabajos antes citados de Feixa (2000; 2006; Leccardi \& Feixa, 20II) ya las generaciones se constituirían a partir de un movimiento social, protagonizado por jóvenes, quienes experimentan juntos un mismo hito marcador. Es entonces, la investigación con sujetos concretos en un contexto determinado lo que diferencia este trabajo de los anteriores. Existe una metodología y estrategias que buscan acercarse a este sujeto joven con nuevas formas organizativas y de comunicación.

En la literatura anglófona se ha vuelto también la mirada sobre la perspectiva generacional, para comprender las sociedades y sus transformaciones. Destaca dentro del enfoque culturalista, el trabajo de Edmunds \& Turner (2005), quienes también identifican el resurgimiento de la perspectiva generacional, a partir de los postulados de Mannheim. Pero a diferencia de otros trabajos, Edmunds \& Turner (2005) critican la conceptualización de Mannheim, específicamente, por estar pensada dentro de límites nacionales, producto del contexto en el que desarrolla su obra.

Por lo anterior, la propuesta de Edmunds \& Turner (2005) es pensar las generaciones en el contexto actual, marcado por la globalización, ya que desde finales del siglo XX y comienzos del siglo XXI se puede experimentar un evento histórico de manera simultánea, como, por ejemplo, el derrumbe de las Torres Gemelas. Tiene especial rendimiento para pensar eventos traumáticos, que hace se tomen posturas al respecto. Por ello, de acuerdo a los autores, la construcción de una conceptualización sobre las generaciones necesita pensar acerca de la globalización.

En este enfoque los autores y postulados, en general, parten de la revisión de autores clásicos que han abordado la perspectiva generacional. La gran mayoría reconocen en Mannheim (I993) y la conceptualización que desarrolló para comprender las generaciones un aporte relevante y actual. No obstante, comparten una fuerte influencia de la visión de Ortega y Gasset (1966; 1970), en tanto, las generaciones se definen principalmente por la cercanía en la fecha de nacimiento y por compartir ciertas coordenadas histórico-espaciales, esta vez, pensadas en términos de globalización. Pero sobre todo, en la comprensión de la historia como una sucesión de generaciones, como lo plantea Feixa (2006), en tanto, cada ıo años estaríamos en presencia de una nueva generación, con referentes culturales y políticos propios. Evidentemente, que la perspectiva culturalista no considera tal cual la obra de Ortega y Gasset, pero es posible leer su influencia en las concepciones a la base antes mencionadas. Sobre todo, el afán de comprender a la sociedad por medio de los cambios y/o empalmes generacionales, cuestión que puede ser problemática, ya que se corre el riesgo de situar y etiquetar a jóvenes diversos, de contextos diferentes en una misma 
categoría, que más que colaborar en la comprensión de las juventudes, puede nublar la heterogeneidad y complejidad del análisis.

Las metodologías que se utilizan en este enfoque no son mayormente explicitadas, cuestión que se entiende para el género del ensayo y revisión (Leccardi \& Feixa, 2012) pero tampoco se explicita mayormente en estudios que hacen referencia a sociedades y jóvenes en contextos concretos (Feixa, 2006; Portillo et al, 2012), exceptuando el trabajo de Fernández-Planells et al (2013), pero que es sobre movimientos sociales y que hace referencia a una serie de estrategias diversas, que permiten acercarse a los sujetos jóvenes online y offline. Entonces, queda como un desafío la mayor producción de trabajos empíricos, haciendo un fuerte hincapié en la metodología y en las diversas técnicas que pueden ser útiles para acercarse a los y las jóvenes en el contexto actual.

Un aspecto relevante de este enfoque, es que busca comprender las generaciones contemporáneas, a partir de procesos como la globalización. Las juventudes están viviendo situaciones y contextos muy distintos a los de generaciones anteriores, con cambios acelerados en sus formas de relacionarse, identidades, subjetividades y en sus vínculos con las instituciones y estructuras sociales. Por ello, es necesario actualizar la perspectiva generacional, sobre todo pensando en las nuevas condiciones sociales, que desbordan en muchas ocasiones los territorios nacionales y locales.

\section{ENFOQUE HISTÓRICO-SOCIOLÓGICO}

En el enfoque histórico-sociológico, los autores comprenden a las generaciones, teóricamente, a partir de la conceptualización desarrollada por Mannheim, con nociones como: posición generacional, conexión generacional, unidad generacional y estratificación de la vivencia (Mannheim, I993). Mannheim (I993) plantea que las generaciones no necesariamente corresponden a grupos concretos, pero pueden llegar a constituirse como tal, sobre todo a partir de la noción de unidades generacionales. En este sentido, dentro de este enfoque existen algunos trabajos que para estudiar a las generaciones se remiten a indagar en grupos concretos, en tanto, utilizan metodologías en ese sentido, grupos focales, entrevistas y encuestas (Canales \& Ghiardo, 2012; Muñoz, 20IIb; 20I8; Santibáñez \& Ganter, 2016).

En el enfoque histórico-sociológico destacan los trabajos de Ghiardo (2004) y Muñoz (20ı1a; 20ırb; 20I8), que abordan cuestiones teóricas y presentan propuestas para abordar esta perspectiva en el campo específico de los estudios de juventud. A su vez, los desarrollos empíricos de estos autores responden a dos corrientes dentro de este enfoque, por un lado, el campo investigativo de las trayectorias a partir de contextos generacionales particulares y cambiantes (Dávila \& Ghiardo, 2012; Ghiardo, 2004) y por otro, la comprensión de grupos definidos a partir de una cierta problemática histórica y las construcciones subjetivas que realizan los sujetos respecto a dicha problemática (Muñoz, 20ıra; 
20IIb; 20I8; Santibáñez \& Ganter, 20I6) (Ghiardo, 2004).

\section{GENERACIONES: TRAYECTORIAS JUVENILES EN CONTEXTOS CAM- BIANTES}

En la primera corriente, destaca el trabajo de Ghiardo (2004), que inaugura la revisión de la perspectiva generacional en RUD, volviendo la mirada sobre ésta y su importancia. Realiza una lectura de autores clásicos - Ortega y Gasset, Mannheim y Bourdieu-, que han abordado previamente la temática. De esta manera, problematiza cuestiones como la edad y su poder de explicar fenómenos sociales; la relación de la sucesión generacional con el cambio, la teoría de la historia y el tiempo; los modos en que se produce la juventud/es, y las distintas identidades. El autor, a lo largo del texto relaciona ideas conceptuales con informes de encuestas nacionales - PNUD e INJUV-, para mostrar empíricamente aquello que se aborda desde la teoría.

De acuerdo a Ghiardo (2004), siempre que se habla de juventudes se hace en referencia a generaciones y las relaciones inter e intra generacionales. Entonces, el estudio de las juventudes, desde esta perspectiva, permitiría comprender las distintas maneras en que una sociedad produce jóvenes, en un determinado tiempo histórico. Para lo cual, se identifican acontecimientos y procesos históricos que marcan a la generación. Junto con ello, se pueden ir comparando los cambios entre las distintas generaciones a través del tiempo, a lo que se debe agregar clase, espacio y género. En este marco comprensivo se pueden desarrollar dos caminos investigativos: uno, analizar la historia específica de un grupo en distintas dimensiones, por medio de la determinación de acontecimientos y procesos históricos, se puede llegar a construir trayectorias típicas; dos, identificar unidades generacionales - concepto desarrollado por Mannheim- a partir de ideas e identidades que los agrupan, de esta manera, se comparan los distintos modos de producir juventudes en un mismo tiempo histórico y en relación a tiempos pasados.

En un trabajo posterior, Canales \& Ghiardo (2012), estudian empíricamente la relación entre distintas cohortes etarias y aspectos socio-culturales que caracterizan a las décadas del proceso de modernización en Chile - que sitúan desde la década del 50-, analizando la constitución de grupos epocales, a partir de la vivencia directa del momento histórico o por resonancia cultural de éste. Los resultados señalan que a los sujetos los marca lo vivido, por ello, todas las cohortes marcan en el tiempo presente, los jóvenes y adultos no marcan en tiempos que no han vivido. Los sujetos definen su generación a partir del período en el que vivieron su juventud, lo que coincide con las conceptualizaciones teóricas como las de Mannheim. No obstante, sus conclusiones tienen un fuerte influjo de Ortega y Gasset, pues señalan que las sucesiones generacionales explicarían el cambio social, un grupo está saliendo, dos grupos son protagonistas y otro grupo está entrando a escena, así un grupo está por 20 años “en la 
cresta de la ola" (p. 39) y luego va saliendo de escena.

Las conclusiones del estudio de Canales \& Ghiardo (2012) muestran esta sucesión generacional como una explicación de las transformaciones sociales, pero no se especifica cómo ni en qué sentido. Asimismo, persistir en la conceptualización de Ortega y Gasset (i966; I970), en el sentido que lo hacen los autores, corre el riesgo de reificar el ciclo vital como una cuestión natural, sin contexto para comprender los grupos de edad que se estudian. Esta visión implica volver a considerar el factor etario como explicativo de las juventudes y sus modos de pensar y actuar, lectura que más bien se ha ido desestimando en este campo investigativo (Bourdieu, I99I; Duarte, 20I2).

En la línea sobre trayectorias juveniles, el trabajo de Dávila \& Ghiardo (2012), indaga en los modos de articulación de elementos objetivos y subjetivos en los procesos de tránsito a la autonomía y la independencia en las y los jóvenes. Para ello, desarrollan un trabajo teórico con gran influencia del Grupo de Investigación en Educación y Trabajo (GRET), de la Universidad Autónoma de Barcelona. Si bien este artículo no ahonda en la comprensión teórica de generaciones, sí la considera un punto clave para estudiar las transiciones a la adultez por parte de los y las jóvenes.

La propuesta teórica de Dávila \& Ghiardo (2012) es desarrollar un análisis biográfico, entendiendo a la sociedad como la estructura, a hombres y mujeres como agentes, y a las generaciones como resultantes de los procesos de cambio. En este modelo se uniría entonces, estructura, acción e historia. En esta lógica, las transiciones a la vida adulta se pueden comprender a partir de condiciones estructurales y pautas culturales de las distintas generaciones, considerando también clase social y género. Las hipótesis que manejan son: uno, que las diferencias generacionales son mayores en jóvenes de sectores bajos, que en aquellos con padres profesionales. Dos, la flexibilidad laboral afecta en mayor medida a los jóvenes de sectores medios y medios altos, que tienen mayor vínculo con el trabajo estable. Tres, cambios en el tránsito a la vida adulta tiene relación también con transformaciones en los imaginarios sobre género, que es más pronunciado en las clases medias. Cuatro, las diferencias en las tasas de fecundidad tienen bases culturales, intergeneraciones y son distintas dependiendo de la clase social. Este trabajo más que desarrollar una propuesta teórica sobre las generaciones, plantea una serie de cambios en el contexto y en las experiencias juveniles. A partir de lo cual, se puede inferir que las generaciones, se diferencian por el cambio en las condiciones estructurales y por el modo de enfrentarla por parte de los y las jóvenes.

Respecto a esta corriente dentro del enfoque histórico-sociológico, surge el riesgo de perder de vista al sujeto joven, pues la construcción de trayectorias típicas y los modos en los que la sociedad produce jóvenes y juventudes, habla más bien de una comprensión de la sociedad determinada por las estructuras sociales, se pierden de vista los procesos de individuación y la agencia de los sujetos en sus propias trayectorias y construcciones identitarias juveniles. Junto 
con ello, destacar que en términos metodológicos, existen pocas referencias en estos trabajos al respecto, ya sea porque responden más bien a ensayos (Ghiardo, 2004), avances de investigación (Dávila y Ghiardo, 2012), pero aún en las investigaciones empíricas (Canales \& Ghiardo, 2012) se entregan pocas pistas sobre el modo de abordar metodológicamente a las generaciones, lo que es vital en pos de operacionalizar este concepto y avanzar en una mayor producción de trabajos empíricos, de modo tal de historizar las trayectorias de jóvenes en términos generacionales.

\section{GENERACIONES: MILITANCIAS Y SUBJETIVIDADES POLÍTICAS}

En la segunda corriente dentro de este enfoque, están los trabajos de Muñoz (20I1a; 20IIb, 20I8) y Santibáñez \& Ganter (20I6). El primer autor ha desarrollado una larga trayectoria abordando la perspectiva generacional, tanto teóricamente como empíricamente (20Ira; 20IIb, 20I8). Muñoz se centra en el enfoque generacional y sus posibilidades en la comprensión de las juventudes, específicamente de las militancias y subjetividades políticas, para lo cual desarrolla una propuesta epistemológica y teórica, haciendo hincapié en el carácter relacional e identitario en la constitución de imaginarios generacionales, a partir del estudio de experiencias de movimientos socio-políticos en México y Chile.

La propuesta de Muñoz (20Ira; 20IIb) elabora dos puntos claves y problemáticos de la perspectiva generacional, por un lado, la definición teórica de las generaciones; y por otro, los usos para la investigación social de esta perspectiva. En el primer punto, marcado por la conceptualización desarrollada por Mannheim (1993), entiende a las generaciones como construcciones diversas, no como grupo homogéneo, que comparte proximidad en la fecha de nacimiento, pero no es esto lo que las define. Una cohorte no es una generación, existen diferencias sustanciales en su interior (unidades generacionales). La irrupción de distintas generaciones, se daría, a partir de vivencias socio-históricas que experimentan los sujetos y las interpretaciones que realizan de éstas, remitiendo con ello a marcas epocales, memoria e identidad. En esta delimitación conceptual, la relación entre juventud y generación, se da porque este periodo -entendido como dinámico y de manera contextual - se constituye en un momento fundante, que marca la aproximación al mundo social por parte de los sujetos - haciendo referencia al concepto de estratificación de la vivencia de Mannheim.

En el segundo punto, la perspectiva generacional se constituye en una categoría para analizar una determinada problemática socio-histórica, a partir de los múltiples factores que inciden en ella. Es un concepto flexible, que hace referencia al cambio social, a partir del pulso que entrega el nacimiento y la muerte biológica de los sujetos, y la relación de éste con la construcción de las sociedades. En este sentido, esta perspectiva sirve para interrogar y cuestio- 
nar ciertos discursos de poder que sitúan a las generaciones jóvenes en polos opuestos, ya sea como un futuro esperanzador o amenazador. Finalmente, este enfoque se aleja de las concepciones de generación ligadas y ancladas en lo etario, no la desconoce por cierto, pero entiende las edades como dinámicas y contextuales, atravesadas por procesos históricos, que pueden ser estudiados e historizados (Muñoz, 20Ira; 20I8).

En esta misma línea encontramos la investigación de Santibáñez \& Ganter (20I6), quienes también estudian generaciones de jóvenes y política en Chile. Parten de la conceptualización de Muñoz (20ı1a) sobre las generaciones, entendidas como una representación social de un relato y discurso de pertenencia, lejanía y de herencia respecto a otras generaciones. Para ello, identifican ciertos contextos e hitos que serían distintivos entre sí y que marcarían a 3 generaciones políticas. Los hallazgos dan cuenta de elementos que persisten en las generaciones tales como la crítica a los partidos políticos, el rol de la justicia en política, entre otros. Además identifican momentos de ruptura en los imaginarios políticos, como el año 2006 y $20 \mathrm{II}^{4}$, en distintas lógicas. En este sentido, uno de los principales hallazgos, contradice la hipótesis inicial de los autores, los años 2006 y 20II, no marcan dos generaciones distintas sino que son parte de un mismo relato generacional. El primer año como un hito fundante y el segundo como una profundización de éste.

En el contexto internacional, destaca el trabajo de Woodman \& Wyn (20I5a; 20I5b; 20I3; Wyn \& Woodman, 2006), investigadores del Melbourne Youth Research Centre de Australia, quienes han dedicado numerosos trabajos a esta perspectiva y se sitúan en el campo específico de los estudios de juventud. Por lo que se busca identificar puntos de encuentro y diálogos posibles con las perspectivas elaboradas en otros contextos pero autores y teorías a la base coincidentes.

La propuesta de Woodman \& Wyn (2015a; 20I5b; 2013; Wyn \& Woodman, 2006) es pensar las transiciones y las culturas juveniles a través de los lentes generacionales y biográficos, para lo cual realizan una síntesis de las teorías desarrolladas por Mannheim, Bourdieu y Beck, elaborando el concepto de generación social, que se hace cargo de las condiciones socio-históricas específicas y de elementos subjetivos (Furlong et al, 20II).

La propuesta de Woodman \& Wyn (20I5a; 20I5b; 20I3; Wyn \& Woodman, 2006) de mirar a las juventudes en el contexto de la sociedad actual desde los lentes generacionales, busca salir de algunas de las trampas típicas de las ciencias sociales y de los distintos enfoques dentro de esta perspectiva, es decir, centrarse en las estructuras o en las subjetividades. Así, por un lado, critican los enfoques centrados en las trayectorias por la excesiva centralidad de las estructuras, con lo cual se deja de mirar al sujeto joven. Esta corriente la he situado en el enfoque histórico-sociológico, ya que entrega un contexto a

4 Años en que se producen grandes movilizaciones estudiantiles en Chile. 
ciertas trayectorias, analizando cambios y continuidades (Dávila \& Ghiardo, 2012; Ghiardo, 2004). Mientras que el enfoque culturalista es criticado porque pierde de vista elementos estructurales como clase, género y etnia, entre otros. Finalmente, respecto al enfoque histórico-sociológico, Woodman \& Wyn (2015a) en el desarrollo de su perspectiva teórica, se distancian de Manheimm porque la conceptualización que desarrolla está pensada para generaciones políticas, que es precisamente el centro del trabajo de Muñoz (20Ira, 20Irb; 20I8) y Santibáñez \& Ganter (2016). El obejtivo de Woodman \& Wyn 20I5a; 20I5b; 2013; Wyn \& Woodman, 2006) es comprender a las generaciones no sólo como un grupo que desarrolla una conciencia política particular, de modo tal que pueda ser una herramienta conceptual para la investigación de campos como educación, salud y trabajo en los estudios de juventud. Para ello, incorporan a autores como Bourdieu y Beck, que permitirían dar cuenta de elementos más cotidianos.

\section{DIÁLOGOS, PRECAUCIONES Y DESAFÍOS}

La perspectiva generacional, de acuerdo a Leccardi \& Feixa (20II), está plenamente vigente en los estudios de juventud, pero como crítica sostienen que no tiene nuevos desarrollos teóricos ni metodológicos. A partir del ejercicio aquí realizado pondré a prueba esta afirmación.

Los autores de base de los distintos enfoques y corrientes son Ortega y Gasset (1966; 1970) y principalmente Mannheim (1993). Este último, escribió en 1928 "El problema de las generaciones", texto citado transversalmente por todos los trabajos que abordan la perspectiva generacional. ¿Por qué la vigencia de este autor? Al respecto, Muñoz (20IIa; 20I8) plantea que se debe al aporte de una perspectiva que problematiza la comprensión de las generaciones, las edades, la juventud y el cambio social. La lectura de Muñoz (20ıra; 20ırb; 20I8) es específicamente sobre la utilidad de esta perspectiva para las militancias políticas, ya que permite unir historia social y política, y estudiar la construcción de imaginarios políticos, vinculado a elementos subjetivos y objetivos en la construcción de un nosotros generacionales y un otro. En definitiva, es una herramienta teórica útil mientras sea siempre para complejizar la comprensión del objeto de estudio en cuestión.

A lo anterior, agregaría que el trabajo de Mannheim fue descomponer el concepto de generación en varias categorías analíticas: posición generacional, unidades generacionales, estratificación de la vivencia, situación de generación y conexión generacional; que permiten de cierto modo operacionalizarlo, y por ende, lo hacen posible de investigar por las ciencias sociales. Esto último, resulta de vital importancia, sobre todo considerando la escasa referencia a las metodologías empleadas en los distintos enfoques y corrientes que abordan lo generacional.

Es claro el consenso en el aporte de Mannheim, para comprender las ge- 
neraciones en la actualidad. Sin embargo, también es evidente la influencia de Ortega y Gasset (i966; i970) Así, en el enfoque culturalista, sigue pesando de manera muy fuerte, la idea de generación desarrollada por el filósofo español, sobre todo, en la obra de Feixa (2000, 2006), autor central en este enfoque, quien comprende de la historia del siglo XX como una sucesión de generaciones, las que a su vez son definidas en cada década y rotuladas con alguna letra. Mientras que en el enfoque histórico-sociológico, la obra de Mannheim (I993) es central, sobre todo aquellos conceptos que refieren a la conformación de identitades más cohesionadas (conexión y unidad generacional), en relación a un contexto histórico particular.

Uno de los riesgos de trabajar con la perspectiva generacional es que es muy probable caer en la definición de patrones generales, es decir, hablar de generaciones como una cohorte de contemporáneos que comparte valores y experiencias homogéneas (Furlong et al, 20II). No todos los y las jóvenes presentan las características propias de una generación, muchos quedan excluidos de las definiciones que la caracterizarían. Este enfoque puede ser poco sensible a quienes quedan en los márgenes (Furlong et al, 20II). Parece ser que cuando se nombra a una generación, se cierra su entendimiento, se define como algo homogéneo aunque se declare previamente que se comprenden las diferencias, ya sea por género, territorio, etnia, etc.

La perspectiva generacional puede constituirse en un aporte para la comprensión de procesos macro sociales en relación con las experiencias, pero, para ello, según France \& Roberts (20I4), necesita de mayor desarrollo teórico y empírico. Esta última afirmación se complementa con lo señalado por Leccardi \& Feixa (20I2), sobre el poco trabajo teórico y metodológico en esta perspectiva. Volviendo sobre estas dos afirmaciones es posible señalar que:

- El trabajo teórico, en general, recurre a autores clásicos. No obstante, se observa en los distintos enfoques una apuesta por actualizar estas primeras teorizaciones. En el enfoque culturalista, se busca incorporar en la conceptualización el proceso de globalización; principalmente, lo referido a los cambios culturales y su relación con las juventudes contemporáneas. Mientras que en el enfoque histórico-sociológico, es central la teoría de Mannheim (I993), y se actualiza con la incorporación de autores y corrientes contemporáneos.

- Las metodologías de los distintos enfoques son un punto escasamente abordado. Por una parte, el enfoque culturalista, tiene artículos que más bien corresponden a ensayos (Feixa, 2000; 2006) y los trabajos empíricos que existen entregan pocas pistas sobre el modo en el que desarrollaron sus investigaciones (Portillo et al, 2012). Por su parte, el enfoque histórico-sociológico, estudia grupos concretos, teniendo como método preferente, lo cualitativo, por medio de técnicas como entrevistas, grupos 
focales. No se observa el desarrollo de metodologías y técnicas preferentes para la perspectiva generacional, en relación a la conceptualización a la base de los distintos enfoques.

- En términos de investigación social aplicada, es posible sostener, que aún existe poco trabajo en este sentido. Desde el resurgimiento de esta perspectiva, los esfuerzos han estado más bien centrados en revistar a autores clásicos, ver de qué manera sus conceptos y teorías pueden ser herramientas útiles para la investigación en juventudes. En esta revisión, el enfoque histórico-sociológico ha desarrollado mayor cantidad de investigación empírica, sobre todo, en términos de militancias y subjetividades políticas, lo que tiene relación con la continuidad de una línea de mayor desarrollo en Chile (Baño y Faletto, I992; Contreras y Navia, 2013; Madrid, 2005). Por lo mismo, es de esperar que en el marco de los estudios sociológicos y culturalistas se avance hacia una mayor producción de conocimiento, lo cual, a la vez va a permitir un mayor desarrollo teórico y metodológico, en la medida que esas epistemes se pongan en relación con sujetos y contextos concretos.

Esta revisión de estudios que trabajan con la perspectiva generacional tiene algunas limitaciones. Si bien RUD es una publicación que acepta aportes de distintos países, aun así, es notoria la mayor preponderancia de autores e investigaciones chilenas. El enfoque culturalista tiene mayor aporte de autores latinoamericanos y españoles. Se constituye como un desafío para la revista el buscar un diálogo más sistemático con autores y corrientes de otras latitudes, como una forma de enriquecer las perspectivas teóricas y metodológicas.

\section{REFERENCIAS}

Baño, R. y Faletto, E. (1992). El apoliticismo:factor generacional. Santiago de Chile: Documentos FLACSO.

Canales, M. \& Ghiardo, F. (2012). Épocas, cohortes y generaciones de chilenos/as: exploración en torno a los grupos socioepocales. Última Década, 2o(36), II-4I. https://dx.doi.org/ro.4067/So718-22362012000I00002

Canales, M.; F. Ghiardo \& A. Opazo (2015) Para un concepto de juventud. En P. Cottet (Ed.) Fuventudes: metáforas del Chile contemporáneo (pp. 47-67). Santiago: RIL editores.

Contreras, G. \& Navia, P. (2013). Diferencias generacionales en la participación electoral en Chile, I988-2010. Revista de Ciencia Política, 33(2), 419-44I.

Dávila, Ó. \& Ghiardo, F. (2012). Transiciones a la vida adulta: generaciones 
y cambio social en Chile. Última Década, 20(37), 69-83. https://dx.doi. org/ro.4067/So718-22362012000200004

Duarte, C. (2005) Trayectorias en la construcción de una sociología de lo juvenil en Chile. Revista Persona y Sociedad, XIX(3), I63-182.

Duarte, C. (2012). Sociedades adultocéntricas: sobre sus orígenes y reproducción. Última Década, 2o(36), 99-125. https://dx.doi.org/ro.4067/So71822362012000100005

Duarte, C. (2015). Estudios juveniles en Chile: devenir de una traslación. En P. Cottet (Ed.). Fuventudes: metáforas del Chile contemporáneo (pp. 23-45). Santiago: RIL editores.

Edmunds, J. \& Turner, B. S. (2005), Global generations: social change in the twentieth century. The British fournal of Sociology, 56 (4): 559-577. DOI:Io.III $\mathrm{I} / \mathrm{j} . \mathrm{I} 468-4446.2005 .00083$.

Feixa, C. (2000). Generación @ la juventud en la era digital. Nómadas, Q 75-9I.

Feixa, C. (2006). Generación XX. Teorías sobre la juventud en la era contemporánea. Revista Latinoamericana de Ciencias Sociales, Niñez y fuventud , 4(2), 2I45 .

Fernández-Planells, Ariadna, Feixa Pampols, Carles, \& Figueroas-Maz, Mònica. (2013). I5-M En España: Diferencias y Similitudes en las Prácticas Comunicativas con los Movimientos Previos. Ultima década, 2I(39), II5I38. https://dx.doi.org/Io.4067/So718-223620I30002000o6

France, A. \& Roberts, S. (20I5). The Problem of Social Generations: A Critique of the New Emerging Orthodoxy in Youth Studies. Fournal of Youth Studies, I8 (2): 215-230. DOI: I0.I080/ I367626r.20I4.944II22

Furlong, A. , Woodman, D. \& Wyn, J. (20II) Changing times, changing perspectives: reconciling 'transition' and 'cultural' perspectives on youth and young adulthood. Fournal of Sociology, 47(4), 355370. DOI:IO.II77/I4407833II420787.

Furlong, A. \& Cartmel, F. (2007) Young People and Social Change: New Perspectives. 2nd ed. Maidenhead: Open University Press.

Ghiardo, F. (2004). Generaciones y Juventud: una Relectura desde Manheim y Ortega y Gasset. Última Década, I2(20), II-46. https://dx.doi. org/I0.4067/So718-22362004000100002

González, Y. \& Feixa, C. (2013). La juventud en el siglo XX: metáforas generacionales. En Y. González \& C. Feixa. La construcción histórica de la Juventud en América Latina. Bohemios, Rockanroleros y Revolucionarios (pp. 75-II9). Chile: Cuarto Propio.

Infantino, J. (2013). La Cuestión Generacional desde un Abordaje Etnográfico: Jóvenes Artistas Circenses en Buenos Aires. Última Década, 2I(39), 87II3. https://dx.doi.org/I0.4067/So718-22362013000200005

Leccardi, C. \& Feixa, C. (20II). El concepto de generación en las teorías sobre la juventud. Última Década, I9(34), II-32. https://dx.doi.org/Io.4067/ So718-22362011000100002 
Madrid, S. (2005) • ¿Políticos de ayer, apáticos de hoy? Generaciones, juventud y política en Chile. En Fuentes, C. \& Villar, A. (Eds.) Voto ciudadano. Debate sobre la inscripción electoral (pp. 45-84). Santiago: FLACSO.

Mannheim, K. (I993). El problema de las generaciones. REIS: Revista española de investigacioes sociológicas. (62), 193-244.

Muñoz, V. (20Ira). Juventud y política en Chile: Hacia un enfoque generacional. Última Década, I9(35), II3-I4I. https://dx.doi.org/Io.4067/So7ı22362011000200006

Muñoz, V. (20IIb). Generaciones. Fuventud universitaria e izquierdas políticas en Chile y México. (Universidad de Chile-UNAM Ig84-2006). Santiago de Chile: LOM Ediciones.

Muñoz, V. (2018). La cuestión generacional en el discurso del gremialismo y la Unión Demócrata Independiente durante la dictadura de Pinochet. Revista de historia y geografia, (39). Ediciones UCSH.

Ortega y Gasset, J. (I966). La idea de generaciones. En El tema de nuestro tiempo, Obras Completas, III (), (pp. I45-I68). Madrid: Revista de Occidente,

Ortega y Gasset, J. (1970). El método de las generaciones. En En torno a Galileo. Obras completas, $I V \emptyset$, (pp. II-7I). Madrid: Revista de Occidente.

Portillo, Maricela, Urteaga, Maritza, González, Yanko, Aguilera, Óscar, \& Feixa, Carles. (2012). De la generación X a la generación @.: trazos transicionales e identidades juveniles en América Latina. Última Década, 20(37), I37-I74. https://dx.doi.org/ro.4067/So718-22362012000200007

Santibáñez, P. \& Ganter, R. (20I6). Representaciones sociales de lo político: Convergencias y divergencias del relato generacional en el gran Concepción. Última Década, 24(44), 39-7o. https://dx.doi.org/ro.4067/So7ı22362016000100003

Woodman, D. \& Wyn J. (20I5a). Youth and Generation. Rethinking change and inequality in the lives of young people. Los Angeles: SAGE.

Woodman \& Wyn (2015b). Class, gender and generation matter: using the concept of social generation to study inequality and social change. Fournal of Youth Studies, I8 (Io), I402-I4Io. DOI: I0.I080/13676261.20I5.IO48206

Woodman, D. \& Wyn, J. (2013). Youth Policy and Generations: Why Youth Policy Needs to 'Rethink Youth'. Social Policy and Society, I2(2), 265-275. DOI: IO.IOI7/Si 474746412000589

Wyn, J. \& Woodman, D. (2006) Generation. youth and social change in Australia. Fournal of Youth Studies, 9 (5), 495 - 514. DOI ro.1080/1367626060о805713 